\title{
The Diurnal Oscillation of MAP (Mitogen-Activated Protein) Kinase and Adenylyl Cyclase Activities in the Hippocampus Depends on the Suprachiasmatic Nucleus
}

\author{
Trongha H. Phan, ${ }^{1}$ Guy C.-K. Chan, ${ }^{1}$ Carlos B. Sindreu, ${ }^{1}$ Kristin L. Eckel-Mahan, ${ }^{2}$ and Daniel R. Storm ${ }^{1}$ \\ ${ }^{1}$ Department of Pharmacology, School of Medicine, University of Washington, Seattle, Washington 98195-7750, and '2Department of Pharmacology, School \\ of Medicine, University of California, Irvine, California 92697-4625
}

\begin{abstract}
Consolidation of hippocampus-dependent memory is dependent on activation of the cAMP/Erk/MAPK (mitogen-activated protein kinase) signal transduction pathway in the hippocampus. Recently, we discovered that adenylyl cyclase and MAPK activities undergo a circadian oscillation in the hippocampus and that inhibition of this oscillation impairs contextual memory. This suggests the interesting possibility that the persistence of hippocampus-dependent memory depends upon the reactivation of MAPK in the hippocampus during the circadian cycle. A key unanswered question is whether the circadian oscillation of this signaling pathway is intrinsic to the hippocampus or is driven by the master circadian clock in the suprachiasmatic nucleus (SCN). To address this question, we ablated the SCN of mice by electrolytic lesion and examined hippocampus-dependent memory as well as adenylyl cyclase and MAPK activities. Electrolytic lesion of the SCN $2 \mathrm{~d}$ after training for contextual fear memory reduced contextual memory measured 2 weeks after training, indicating that maintenance of contextual memory depends on the SCN. Spatial memory was also compromised in SCN-lesioned mice. Furthermore, the diurnal oscillation of adenylyl cyclase and MAPK activities in the hippocampus was destroyed by lesioning of the SCN. These data suggest that hippocampus-dependent long-term memory is dependent on the SCN-controlled oscillation of the adenylyl cyclase/MAPK pathway in the hippocampus.
\end{abstract}

\section{Introduction}

Hippocampus-dependent memory is initiated by $\mathrm{Ca}^{2+}$ signals originating from activation of NMDA receptors (Morris et al., 1986; Danysz et al., 1988; Tsien et al., 1996) and is dependent upon de novo transcription and translation (Davis and Squire, 1984; Tully et al., 1994; Bailey et al., 1996). The transcriptional pathway most strongly implicated in consolidation of hippocampus-dependent long-term memory is the CRE (cAMP/Ca ${ }^{2+}$ response element)-transcription pathway (Bourtchuladze et al., 1994; Athos et al., 2002; Pittenger et al., 2002). One of the key $\mathrm{Ca}^{2+}$-activated pathways for stimulation of CRE-mediated transcription in hippocampal neurons during memory formation is the Erk/MAPK (mitogen-activated protein kinase) pathway (Atkins et al., 1998; Blum et al., 1999; Athos et al., 2002). Activation of Erk/MAP kinase and its nuclear translocation during memory formation depends upon a cAMP signal generated by the calmodulin-stimulated adenylyl cyclases (Sindreu et al., 2007).

Because a single round of transcription and translation cannot account for the persistence of memory over extended periods of

Received Dec. 14, 2010; revised June 3, 2011; accepted June 7, 2011

Author contributions: T.H.P. and K.L.E.-M. designed research; T.H.P., G.C.-K.C., and C.B.S. performed research; D.R.S. contributed unpublished reagents/analytic tools; T.H.P. analyzed data; T.H.P. and D.R.S. wrote the paper.

This research was funded by NIH Grants NS 020498 and MH 073601 to D.R.S. and NIH Neurobiology Training Grant 5 T32 GM07108 to T.H.P. We thank Dr. Horacio de la Iglesa for helpful suggestions concerning the SCN lesioning procedure. We also thank Dr. Jeansok Kim for allowing us to use the lesion maker. We thank members of the Storm lab for critical reading of this manuscript.

Correspondence should be addressed to Dr. Daniel R. Storm, University of Washington School of Medicine, Department of Pharmacology, 1959 NE Pacific Street, HSB Building, Room J681, Seattle, WA 98195-7750. E-mail: dstorm@u.washington.edu.

DOI:10.1523/JNEUROSCI.6535-10.2011

Copyright $\odot 2011$ the authors $\quad 0270-6474 / 11 / 3110640-08 \$ 15.00 / 0$ time, we considered the possibility that the biochemical pathways underlying memory consolidation may be reactivated repeatedly to sustain levels of proteins required for persistence of memory. Recently, we discovered that adenylyl cyclase and Erk/MAP kinase activities undergo a circadian oscillation in the hippocampus that is paralleled by changes in Ras activity as well as the phosphorylation of MEK and CREB (Eckel-Mahan et al., 2008). Circadian oscillations in MAP kinase activity are absent in memory-deficient, transgenic mice lacking $\mathrm{Ca}^{2+}$-stimulated adenylyl cyclases. Furthermore, exposure to constant light, days after training, disrupts both hippocampal oscillations of Erk/ MAPK and contextual memory persistence. Moreover, pharmacological inhibition of MAPK activity at the peak of the cycle, but not the trough, impairs memory persistence. These observations support the hypothesis that the persistence of long-term memories may depend upon reactivation of the cAMP/MAPK/CREB transcriptional pathway in the hippocampus during the circadian cycle. Since the oscillation in this signaling pathway is circadian in nature and persists even under free-running [constant darkness (d/d)] conditions, it was important to determine whether activation of this pathway is intrinsic to the hippocampus or driven by the SCN. Although the SCN plays a major role in maintaining circadian rhythms in animals (Rosbash and Hall, 1989; Reppert and Weaver, 2001; Hastings et al., 2003; Gerstner and Yin, 2010), there are peripheral oscillators in other tissues, including the olfactory bulb (Granados-Fuentes et al., 2004a,b) and the hippocampus, where the circadian genes Perl (Wang et al., 2009), and Bmal1 (Jilg et al., 2010) continue to oscillate, even in the absence of a functional SCN. Here, we report that electrolytic 

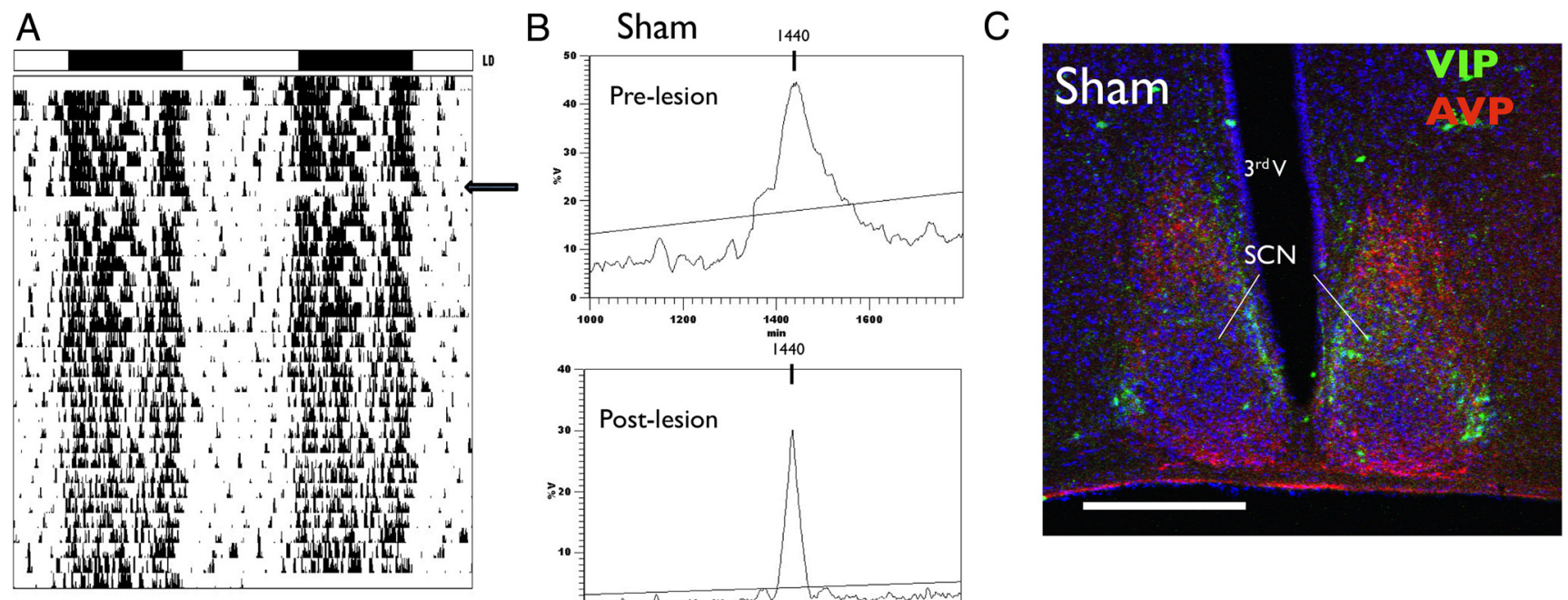

D

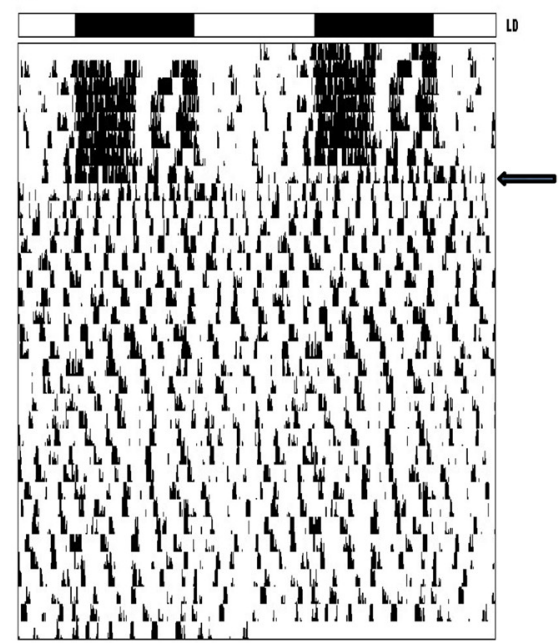

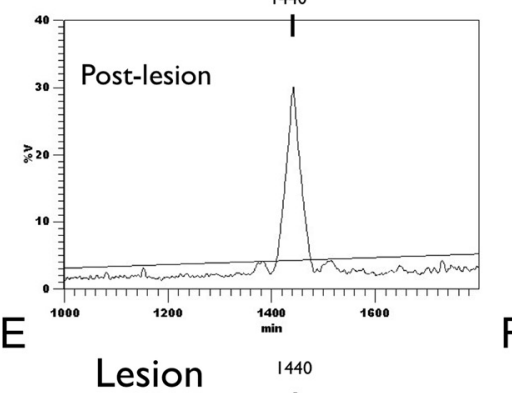
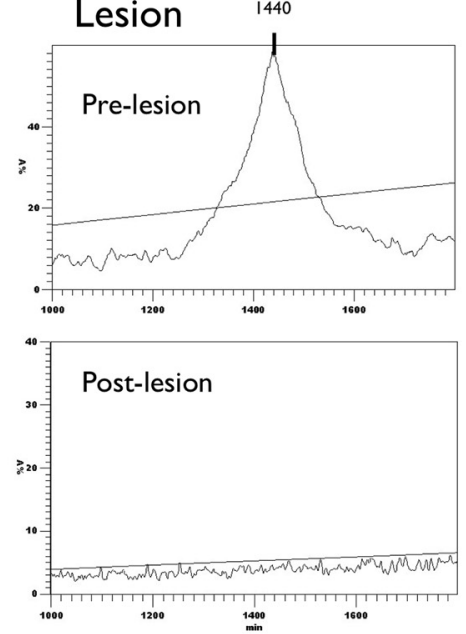

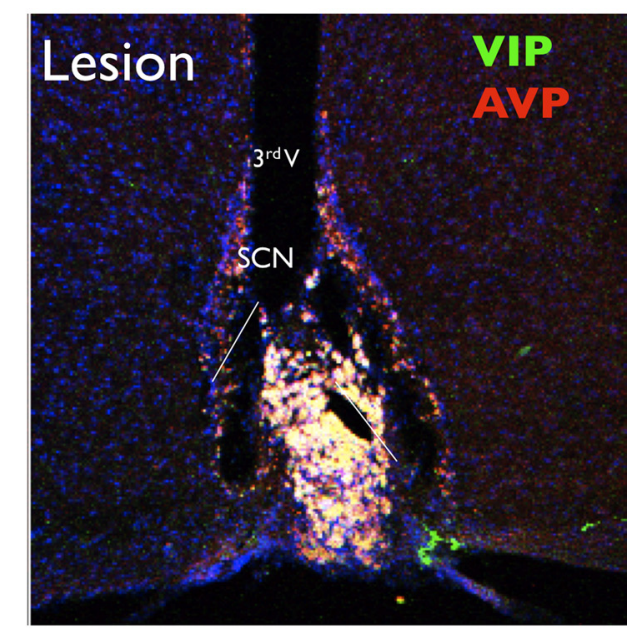

Figure 1. Electrolytic lesioning of the SCN makes mice arrhythmic. $A$, Representative actogram of a sham-lesioned mouse. White and black segments in the top bar indicate hours in light and dark conditions, respectively. Arrow indicates the date the animal was under surgery. $\boldsymbol{B}, \chi^{2}$ periodogram analysis shows that the period, $\tau$, is 1440 min or $24 \mathrm{~h}$ for both pre-sham (top) and post-sham (bottom) lesion under a $12 \mathrm{~h} \mathrm{L/D}$ cycle. Note: $\chi^{2}$ analysis was calculated over $7 \mathrm{~d}$ before surgery, and $28 \mathrm{~d}$ after surgery, which accounts for the sharper peak in the latter. $\boldsymbol{C}$, Representative brain section of a mouse receiving sham-surgery and counterstained with Hoescht (blue), VIP (red), and AVP (green). The SCN was intact. $3^{\text {rd }}$ V, Third ventricle. Scale bar, $200 \mu m$. D, Actogram of an SCN-lesioned mouse showing that the diurnal locomotor activity was intact before lesion; however, no rhythmicity was observed after lesioning of the SCN. The arrow marks the date of SCN lesion. $\boldsymbol{E}, \chi^{2}$ periodogram analysis showing diurnal locomotor activity was intact before lesion, $\tau=24 \mathrm{~h}$ (top); however, no periodicity was observed after lesioning of the $S C N$ (bottom). $\boldsymbol{F}$, Representative brain section of a mouse receiving an electrolytic lesion to the SCN, stained with Hoescht (blue), VIP (red), and AVP (green). Note the degeneration of SCN tissue.

destruction of the SCN destroys the circadian oscillation of adenylyl cyclases and Erk/MAPK activity in the hippocampus, and compromises spatial and contextual memory. Interestingly, destruction of the SCN $2 \mathrm{~d}$ after acquisition of contextual memory destroys memory persistence. These data indicate that oscillation of the cAMP/MAPK pathway in the hippocampus, which is required for the maintenance of hippocampus-dependent memory, is driven by the SCN.

\section{Materials and Methods}

Mice. Adult, male C57BL/6 mice aged 3-5 months were used for all experiments. Experiments were performed in accordance with the Institutional Animal Care and Use Committee's recommendations at the University of Washington.

SCN lesioning. Mice aged from 3 to 4 months were anesthetized with a $20-22 \mu \mathrm{l} / \mathrm{g}$ body weight mixture of ketamine $(7 \mathrm{mg} / \mathrm{ml})$ and xylazine $(0.44 \mathrm{mg} / \mathrm{ml})$. The animals were carefully placed on the stereotaxic frame (Cartesian Research) and secured with two ear bars. Artificial tears ointment (The Medicine Shoppe) was applied to avoid drying of the eyes. The scalp hair was removed and an incision was made at midline. Bregma and lambda lines were located on the skull, which was drilled at the following coordinates: mediolateral (ML), $\pm 0.17 \mathrm{~mm}$; anteroposterior (AP), 0.17 $\mathrm{mm}$; and dorsoventral (DV), $5.55 \mathrm{~mm}$; and an electrode (MicroProbes) that was coated with epoxy (except for $300 \mu \mathrm{m}$ at the tip), was lowered to the target site. An electric current of $1 \mathrm{~mA}$ was delivered by a lesionmaking device (Ugo Basile) for $20 \mathrm{~s}$. The same procedure was performed on the other hemisphere. The mortality rate following SCN lesion was $\sim 20 \%$. For sham surgery, the same procedure was carried out; however, no electric current was delivered. For hippocampus control lesions, the following target was used: ML, $\pm 1.5 \mathrm{~mm}$; AP, $-1.70 \mathrm{~mm}$; DV, $1.65 \mathrm{~mm}$. The animals were sutured and allowed to recover for 2 weeks before any behavioral analysis was carried out.

Contextual memory training. Animals were gently handled for $2 \mathrm{~min}$ at least $5 \mathrm{~d}$ before the experiments. On the day of training, mice were placed in the contextual training chamber, $10(\mathrm{~W}) \times 10(\mathrm{D}) \times 16(\mathrm{H})$ inches. The metal floor consisted of a metal grid with each beam 0.6 inch apart. The mice were placed in the arena for $2 \mathrm{~min}$ to explore the chamber, after which a shock of $0.7 \mathrm{~mA}$ for $2 \mathrm{~s}$ was delivered through the grid. The 


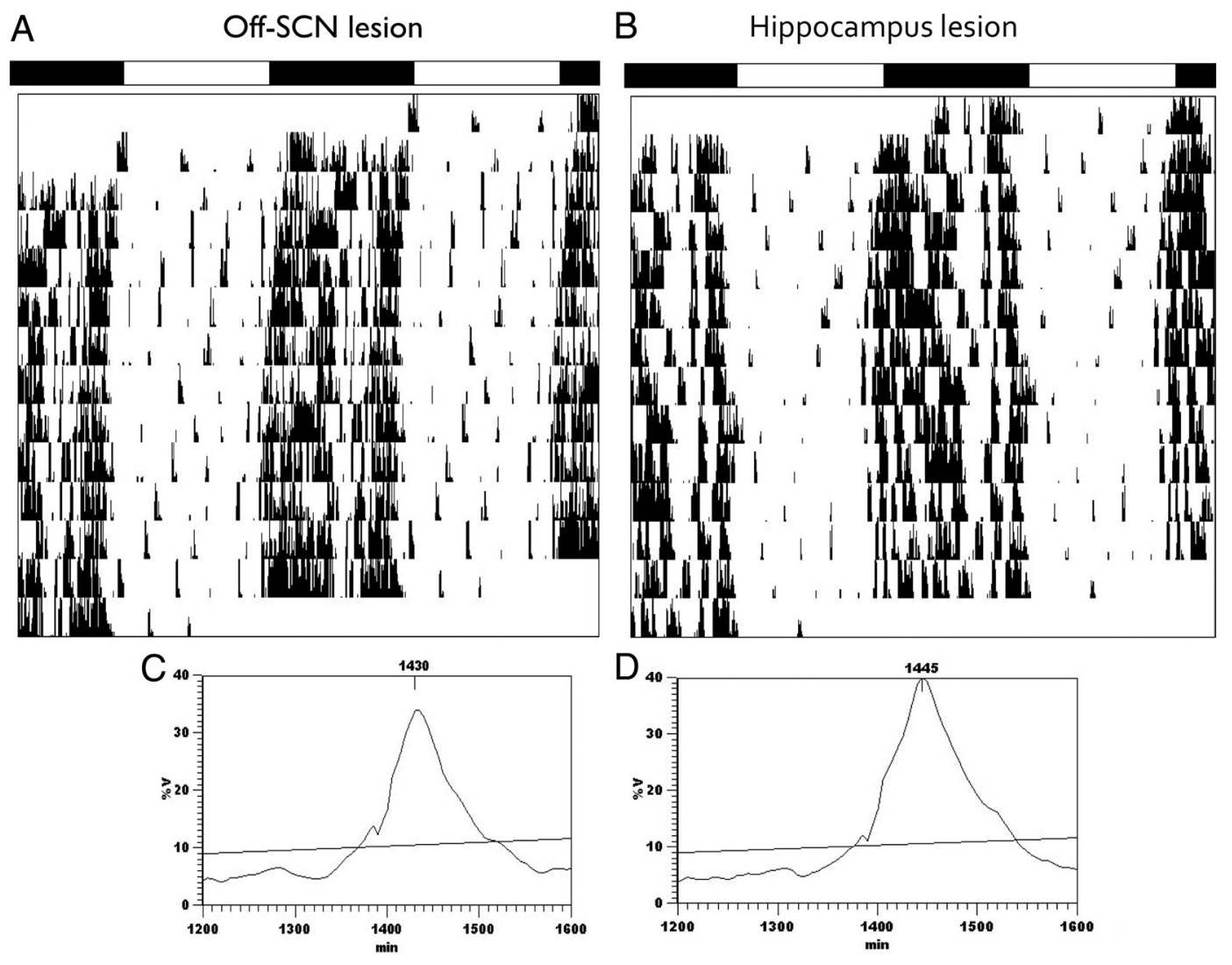

Figure 2. Electrolytic lesion of other areas of mouse brain does not affect circadian rhythmicity. $A$, Actogram of an "off-SCN" target lesioning, made $\sim 1 \mathrm{~mm}$ dorsal to the target site. $\boldsymbol{B}$, Actogram of intact circadian locomotor activity after electrolytic lesions of a small region of CA1 of the hippocampus. $\boldsymbol{C}, \chi^{2}$ periodogram of the off-target lesioning showed a normal $\tau=24 \mathrm{~h}$. $\boldsymbol{D}, \chi^{2}$ periodogram demonstrates that lesioning a small part of the CA1 region of the hippocampus did not affect the animal's diurnal activity rhythms.

animals remained in the chamber for one more minute before returning to their home cages. The metal grid floor and the Plexiglas wall were wiped down with diluted acetic acid after each mouse left the chamber. Two weeks after the training, the mice were placed back into the chamber for $2 \mathrm{~min}$. The amount of freezing (no movements except for breathing) was scored every $5 \mathrm{~s}$. The experiments were videotaped and later scored by investigators who were blind to the conditions for confirmation. The freezing percentage was also confirmed by automated measurement of movement monitored by TruScan (Coulbourn Instruments).

Morris water maze training. The circular pool used for Morris water maze training was $84 \mathrm{~cm}$ in diameter and the escape platform diameter was $5 \mathrm{~cm}$, giving a ratio of the escape platform area to pool area similar to others reported in the literature (Vorhees and Williams, 2006). The pool was filled with tap water and equilibrated to room temperature $\left(\sim 22^{\circ} \mathrm{C}\right)$. The water was mixed with a nontoxic tempura paint to make the water opaque. The animals were placed in the pool at randomized locations designated N, S, E, and W. Each animal had 90 s to find the nonvisible platform just below the surface of the water. Animals that could not find the platform in $90 \mathrm{~s}$ were gently guided to the platform, where they were allowed to recover for $30 \mathrm{~s}$. Each mouse was trained 4 times for 4 consecutive days. The intertrial period was $30 \mathrm{~min}$. The mice were dried off with towels and placed back in their home cages. Each trial was recorded with a digital video camera. The pool location was fixed relative to distal cues surrounding the pool. During the probe trial on day 5 , the platform was removed from the pool and the mice were allowed to search for the target platform for $90 \mathrm{~s}$. The movie files were converted to MPEG file format and analyzed with EthoVision (Noldus). EthoVision tracks the animal movement by subtracting the intensity from background.

Novel object recognition. Mice were placed in rat cages overnight to habituate with the novel environment. On the day of testing, mice were divided into two groups: one group of animals was presented with two identical objects (A1 vs A2), while the other half of the group was pre- sented with another pair of objects (B1 vs B2), one on each side of the cage. Mice were exposed to these objects twice for $5 \mathrm{~min}$, with a $10 \mathrm{~min}$ intertrial interval. The amount of time exploring the object, defined as the head turned in the direction of the object with the vibrissae within 1 $\mathrm{cm}$ of the object, was recorded and converted to percentage of time exploring each object. The time that animals spent climbing on objects was excluded from the analysis. After $10 \mathrm{~min}$, one of the identical objects was replaced with the new object (A vs B), while the other half received the same pair (B vs A) to counterbalance for any innate preference for specific objects. After $24 \mathrm{~h}$, the animals were tested for memory by exposure to a third object, $\mathrm{C}$ along with one of the original objects to measure $24 \mathrm{~h}$ memory. The amount of time spent exploring each object was scored independently by two investigators.

Adenylyl cyclase activity. Mice were killed at ZT4 (zeitgeber time) and ZT16 under dim red light by cervical dislocation. Hippocampi were homogenized in buffer containing (in mM): 50 Tris $\mathrm{HCl}$, pH 7.4, $2 \mathrm{MgCl}_{2}, 1$ EDTA, 0.5 DTT, and Protease Inhibitor Cocktail (Roche). The homogenate was centrifuged at $1000 \times g\left(4^{\circ} \mathrm{C}\right)$ for $2 \mathrm{~min}$, and pelleted membranes were isolated by high-speed centrifugation $\left(100,000 \times g, 4^{\circ} \mathrm{C}\right)$ for $1 \mathrm{~h}$. Pellets were resuspended in homogenization buffer. Adenylyl cyclase assays were performed with $95 \mu \mathrm{l}$ of membrane homogenate in a total volume of $250 \mu \mathrm{l}$ containing the following (in $\mathrm{mm}$ ): 39.2 Tris $\mathrm{HCl}, \mathrm{pH}$ 7.4, 1 ATP, 1.38 EDTA, 0.21 EGTA, $5.76 \mathrm{MgCl}_{2}, 0.1$ DTT, Protease Inhibitor Cocktail, $1 \beta$-mercaptoethanol, 0.2 IBMX, 0.1\% BSA with trace amounts of $\left[\alpha-{ }^{32} \mathrm{P}\right]$ ATP $(\sim 5.0 \times 106$ counts per reaction, NEN $)$. To normalize data for sample recovery during column steps, 10,000 counts per sample of $\left[{ }^{3} \mathrm{H}\right] \mathrm{cAMP}$ (NEN) and $2 \mathrm{~mm}$ cAMP were included. To measure calcium-stimulated activity, calmodulin (50 U per reaction, Sigma) was added to the assay with $350 \mu \mathrm{M}$ calcium ( $7 \mu \mathrm{M}$ "free" calcium) using the Maxchelator algorithm http://www.stanford.edu/ cpatton/ downloads.htm). The reactions were performed for $15 \mathrm{~min}$ at $30^{\circ} \mathrm{C}$ and stopped with $1.5 \%$ SDS and heating to $100^{\circ} \mathrm{C}$ for $2 \mathrm{~min}$. Samples were 


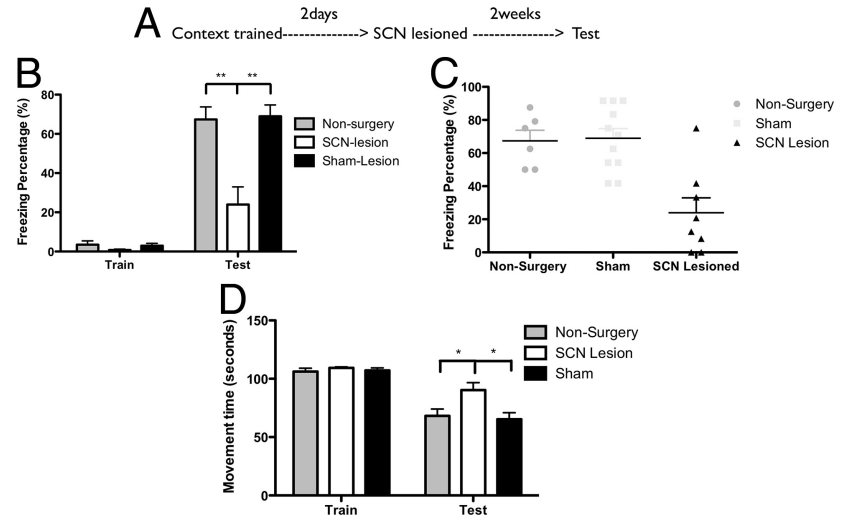

Figure 3. Ablating the SCN $2 \mathrm{~d}$ after training decreases the persistence of hippocampusdependent contextual memory. $\boldsymbol{A}$, The experiment was designed to elucidate the importance of the $\mathrm{SCN}$ for memory maintenance. $\boldsymbol{B}$, The percentage of freezing of the $\mathrm{SCN}$-lesioned group on the testing day is significantly reduced $\left({ }^{* *} p<0.01\right.$, one-way ANOVA, lesion vs other groups). C, The amount of freezing of individual mice during testing. D, SCN-lesioned mice show greater movement during testing. Locomotion was monitored with an automated computer tracking system. Values are expressed as means \pm SEM, $n=6-12$ per group.

Tissue preparation and immunofluorescence staining. Animals were operated on at least 2 weeks before they were killed. One week before they were killed, animals were handled daily for $2 \mathrm{~min}$. At ZT4, ZT12, or ZT16 on the day they were killed, the animals were injected with a ketamine/ xylazine mixture, and transcardially perfused with chilled $4 \%$ formaldehyde and $50 \mathrm{~mm} \mathrm{NaF}$ in $0.1 \mathrm{~m}$ PBS under dim red light. Brains were cryoprotected with $30 \%$ sucrose in $0.1 \mathrm{M}$ PBS, and sectioned to $30 \mu \mathrm{m}$ thickness on a cryostat. Slices remained in cryoprotector solution until staining. Slices were washed with PBS and pretreated with $0.5 \% \mathrm{NaBH}_{4}$ and then in $1 \% \mathrm{H}_{2} \mathrm{O}_{2}$ in $10 \%$ ethanol, followed by blocking in $10 \%$ goat serum, $2 \%$ BSA, $50 \mathrm{~mm}$ glycine, $0.2 \%$ Triton X-100 in PBS for $2 \mathrm{~h}$. The sections were incubated with anti-phospho-ERK Thr ${ }^{202} / \mathrm{Tyr}^{204}$ (cell signaling) 1:10,000 overnight. The cyanine 3-tyramine signal amplification kit (PerkinElmer) was used to optimize p-ERK detection. For detection of vasoactive intestinal peptide (VIP) and arginine vasopressin (AVP), sections were rinsed with TBST (saline $+0.2 \%$ Triton X-100), and blocked in 1\% BSA, $10 \%$ goat serum in TBST for $1 \mathrm{~h}$. Sections were incubated with rabbit anti-VIP (Millipore), 1:250 and guinea pig antiAVP (Bachem), 1:1000, in blocking buffer overnight. Secondary antibodies with conjugated Alexa Fluor 568 (1:500) and 488 (1:500) were incubated for $2 \mathrm{~h}$. Sections were counterstained with Hoescht. Immunohistochemical data were obtained from two rounds of experiments, for a total of 8-9 mice per group. Four hippocampal sections per mouse were analyzed, spanning between $\mathrm{AP}-1.7$ and $-2.3 \mathrm{~mm}$ from bregma. pErk-immunopositive cells in the CA1 pyramidal layer were manually counted throughout the thickness of each section under epifluorescence. Individual values from each section were averaged per mouse, and those in turn were averaged among mice of the same conditions to obtain a final group mean. Counting of sections was performed blind to experimental condition.

Statistical analysis. Reported values are averages for the group \pm SEM. Significance was determined between two groups using parametric Student's $t$ test. ${ }^{\star} p<0.05,{ }^{\star \star} p<0.01$, and ${ }^{* *} p<0.001$. When more than two experimental groups (and with different treatments) were compared, one-way ANOVA or two-way ANOVA was used followed with Bonferroni post hoc test analysis. The number of subjects is designated as $n$.

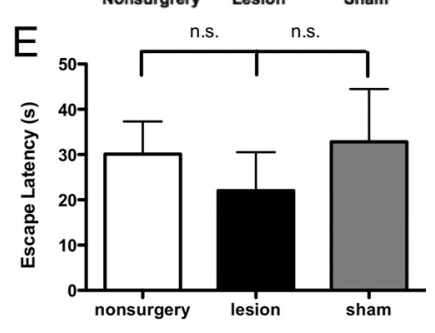

Figure 4. SCN-lesioned mice have impaired spatial memory in the Morris water task. A, Each mouse was trained on four trials per day to find a hidden platform. The time the animals took to reach the platform is reported on the $y$-axis as escape latency. On day 5 , a probe test was administered. On day 6 , a spatial reversal was given to test for working memory. $\boldsymbol{B}$, During the probe trial, the percentage of time the mouse spent in the target quadrant was compared among the groups. The percentage of time that the SCN-lesioned mice spent in the target quadrant was significantly lower than that of non-surgery and sham-lesioned groups (31.66, 45.65 , and $48.25 \%$ respectively, ${ }^{*} p<0.05$, one-way ANOVA, Bonferroni's post hoc test). C, The number of platform crossings was significantly lowered in the SCN-lesioned group compared with the two control groups, ${ }^{*} p<0.05$, one-way ANOVA, Bonferroni's post hoc test. $\boldsymbol{D}$, There was no difference in swim speed among the three groups. $\boldsymbol{E}$, During the reverse spatial training on the sixth day, the SCN-lesioned animals found the platform as fast as the control groups did. Means \pm SEM, $n=7-10$ per group.

cooled and loaded onto ionic, analytical grade, 200-400 mesh columns packed with Dowex AG 50W-X4 resin (Bio-Rad). Samples were eluted onto alumina columns (WN3 type; Sigma) with $\mathrm{ddH}_{2} \mathrm{O}$. A final elution step from the alumina columns was performed with $0.1 \mathrm{M}$ imidazole. cAMP produced was determined by scintillation counting of $\left[{ }^{32} \mathrm{P}\right] \mathrm{cAMP}$ and normalized to $\left[{ }^{3} \mathrm{H}\right]$ cAMP recovered. Protein was determined by the Pierce BCA assay, and adenylyl cyclase activity is reported as pmol of cAMP per minute per mg protein. Blue due to iron deposited from oxidation during current delivery (data not shown). Sham lesion of the SCN did not affect the circadian rhythm (Fig. 1A), while electrolytic lesion of the SCN abolished the circadian rhythm of the mice (Fig. $1 D$ ). The circadian rhythm of the electrolytic lesioned mice was abolished under light/dark (L/D) conditions because the ventral SCN, which receives light input from the retinothalamic tract, was completely 
destroyed (Fig. $1 F$ ). Complete lesioning of the SCN abolishes the circadian rhythm of mice under L/D conditions (Schwartz and Zimmerman, 1991).

To confirm that the arrhythmia was due to specific lesioning of the SCN, tissue was stained for VIP (green), and AVP (red), Hoechst (blue) to verify the location of the lesion (Fig. 1C,F). Any mice that were lesioned outside the SCN were eliminated from behavioral and biochemical analysis. The circadian period, $\tau$, was determined by the $\chi^{2}$ periodogram analysis using a computer program, El Temps (Diez-Noguera, Barcelona, Spain) (Fig. 1B). The horizontal line signifies a 0.05 significance level, and any peak that crosses this line is considered significant. Sham-lesioned animals exhibited a $\tau$ of $24 \mathrm{~h}$ (1440 min) when the animals were on a $12 \mathrm{~h} \mathrm{~L} / \mathrm{D}$ cycle, both before and after sham lesion. However, there was no discernable period in the postlesion of SCN-lesioned animals (Fig. $1 E$ ). Notably, electrolytic lesioning outside the SCN $(>1 \mathrm{~mm}$ outside the SCN) or a small lesion of the hippocampus did not result in arrhythmic animals (Fig. $2 A, B$, respectively). These mice retained their $24 \mathrm{~h}$ period, $\tau=24 \mathrm{~h}$ (Fig. $2 C, D$ ).

\section{Persistence of contextual memory is decreased in SCN-lesioned mice}

Contextual fear conditioning is a form of associative learning that depends on the hippocampus for proper acquisition and expression (Fanselow and Poulos, 2005). Furthermore, persistence of contextual memory is impaired when the oscillation of the cAMP/MAPK pathway in the hippocampus is disrupted (EckelMahan et al., 2008). To address the role of the SCN for the maintenance of contextual memory, we trained animals for contextual fear and then lesioned the SCN $2 \mathrm{~d}$ after training. Two weeks after SCN lesion, animals were placed back into the context, and the amount of time freezing was scored during 2 min testing sessions (Fig. 3A). The SCN-lesioned animals froze significantly less than the non-surgery and sham-lesioned mice during testing $\left(F_{(3,24)}=\right.$ 8.957, $p=0.0004, n=6-10$, one-way ANOVA, ${ }^{\star *} p<0.01$, lesion vs other groups, Bonferroni's post hoc test) (Fig. 3B). The contextual memory of individual mice is displayed in Figure $3 C$. These data indicate that SCN lesioning $2 \mathrm{~d}$ after training significantly decreased the persistence of contextual memory. Similar results were obtained when contextual memory was automatically scored for movement time (Fig. 3D). The movement times for SCN-lesioned animals during testing were significantly higher than those for the sham-lesioned control group $\left(F_{(3,18)}=4.881\right.$, $p=0.0118, n=6-10,{ }^{\star} p<0.05$, lesion vs sham, one-way ANOVA), indicating that persistence of contextual memory was compromised by lesioning of the SCN. Collectively, these results indicate that the $\mathrm{SCN}$ is required for the maintenance of contextual fear memory.

\section{SCN-lesioned mice have impaired spatial memory}

Spatial memory is another form of hippocampus-dependent memory that depends upon MAPK signaling (Blum et al., 1999; Selcher et al., 1999; Li et al., 2005). Therefore, we examined the effect of SCN lesions performed before training for the hidden platform version of the Morris water maze task. Mice were trained four times a day for $4 \mathrm{~d}$. The escape latencies for finding the hidden platform decreased on a daily basis with no differences in learning curves between sham-lesioned and SCN-lesioned mice (Fig. 4A). On day 5, each animal was subjected to a $90 \mathrm{~s}$ probe test in which the hidden platform was removed and the time spent in exploring the original target quadrant and platform area was monitored. Non-surgery and sham-lesioned control groups showed a stronger preference $(45.65 \%$ and $48.25 \%$, re-

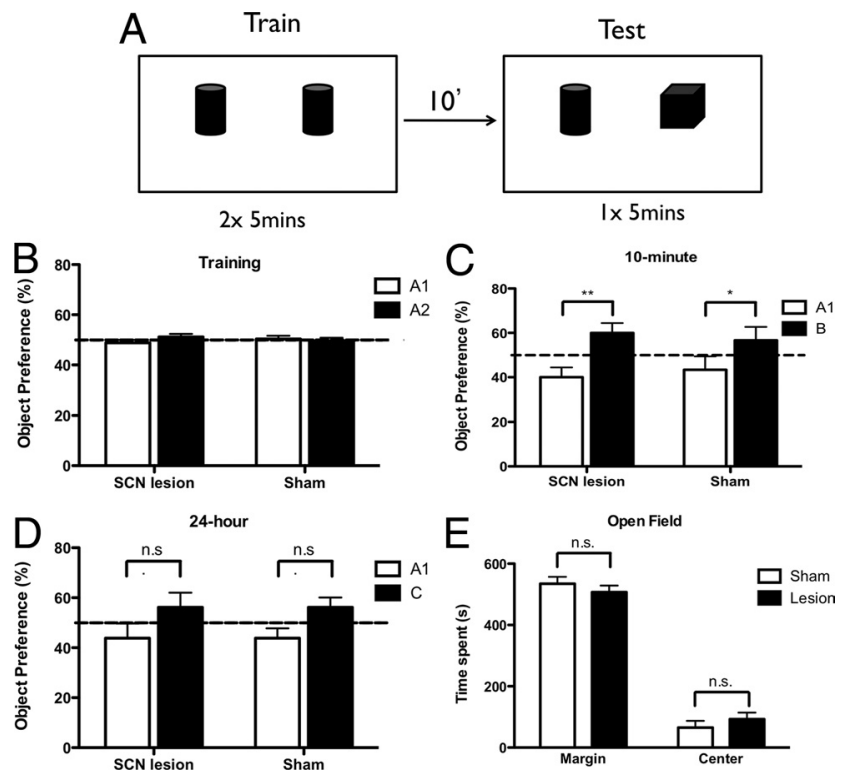

Figure 5. SCN-lesioned mice express normal recognition memory for novel objects and open field test. $A$, Mice were allowed two 5 min periods to examine a pair of identical objects, $A 1$ and $A 2$. During testing, one of the original objects was replaced with a new object, B or C. B , During training, SCNlesioned and sham-lesioned mice spent similar amounts of time exploring each object (50.13 and $51.20 \%)$. C, There was no difference in short-term memory for objects between sham- and SCNlesioned animals measured 10 min after exposure to the original pair of identical objects (lesion, $p=$ 0.0079; sham, $p=0.0491$; two-tailed $t$ tests). $\boldsymbol{D}$, Neither SCN-lesioned nor sham-lesioned mice exhibited long-term memory for objects measured $24 \mathrm{~h}$ after exposure to objects $\mathrm{A} 1$ and $\mathrm{A} 2$. Values are averages of individual animals' performances \pm SEM, $n=7-9$ per group. $\boldsymbol{E}$, The amount of time spent in the center and margin of the arena during an open field test as measured by EthoVision (Noldus) software.

spectively) for the target quadrant compared with the SCNlesioned group $(31.66 \%)$. The time spent in the target quadrant during the probe trial by SCN-lesioned animals was significantly lower than that of the non-surgery group $\left(F_{(3,88)}=35.69, p=\right.$ $<.0001, n=6-10,{ }^{*} p \leq 0.05$, lesions vs non-surgery, two-way ANOVA) or the sham-lesioned group $\left({ }^{* *} p<0.01, n=6-10\right.$, lesions vs sham, two-way ANOVA) (Fig. $4 B$ ). In addition, the total number of platform crossings exhibited by SCN-lesioned mice was significantly lower than that of sham-lesioned mice $\left(F_{(2,24)}=6.4, p=0.0064,{ }^{*} p \leq 0.05\right.$, lesion vs sham, one-way ANOVA) (Fig. 4C). An analysis of search patterns during the probe test showed that SCN-lesioned animals generally made larger searching loops throughout the pool rather than spending time searching the original target area (data not shown). Nevertheless, the SCN-lesioned animals swam with a speed similar to that of the controls ( $p=0.78$, one-way ANOVA) (Fig. $4 D$ ). On day 6 , when the platform was moved to a different location, SCNlesioned mice were able to learn the new location of the hidden platform as quickly as sham-lesioned mice (Fig. 4E). These data indicate that although the SCN-lesioned mice learned to find the hidden platform and showed normal reversal learning, they had poorer spatial memory for the location of the platform.

\section{SCN-lesioned mice exhibit normal memory for novel objects and normal open field behaviors}

To examine the importance of the SCN for other types of memory, SCN-lesioned mice were examined for short-term recognition memory for objects. Mice were exposed to two identical objects twice for $5 \mathrm{~min}$ with a $10 \mathrm{~min}$ intertrial interval (Fig. 5A). During training, sham-lesioned and SCN-lesioned mice did not ex- 


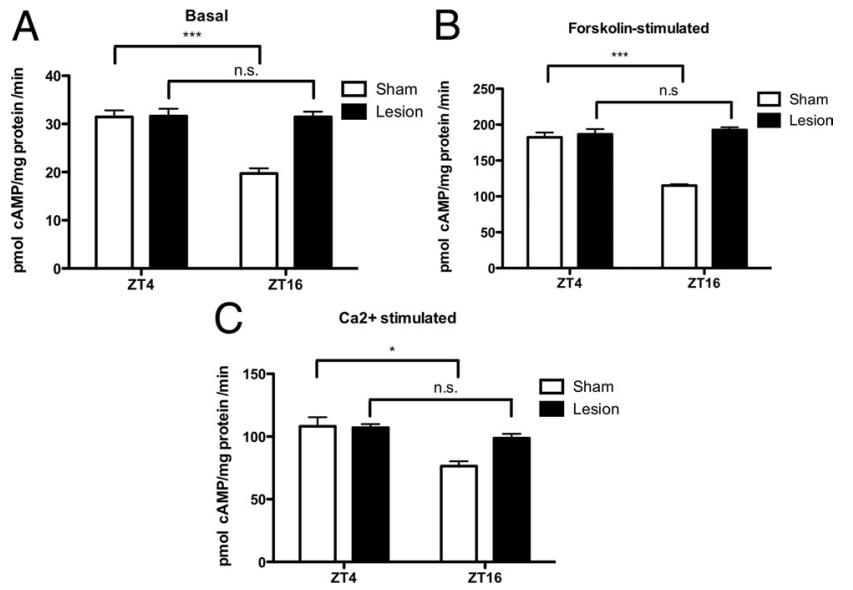

Figure 6. Ablating the $\mathrm{SCN}$ destroys the diurnal oscillation of $\mathrm{Ca}^{2+}$-sensitive adenylyl cyclase activity in the hippocampus. $A$, In contrast to sham-lesioned mice, basal adenylyl cyclase activity in the hippocampus of SCN-lesioned mice did not show a diurnal oscillation. $\boldsymbol{B}$, Forskolin stimulated adenylyl cyclase activity in the hippocampus from sham-lesioned mice, but not $\mathrm{SCN}$-lesioned mice, showed a diurnal oscillation. $\mathrm{C}_{\text {, }} \mathrm{Ca}^{2+}$-stimulated adenylyl cyclase activity in the hippocampus from sham-lesioned mice, but not SCN-lesioned mice, showed a diurnal oscillation. Values are expressed as mean \pm SEM. Experiments were performed using pooled tissue from $4-5$ animals per group, and tissue samples were assayed in triplicate. ${ }^{* *} p<$ $0.001,{ }^{*} p<0.05$, two-way ANOVA, Bonferroni's post hoc test.
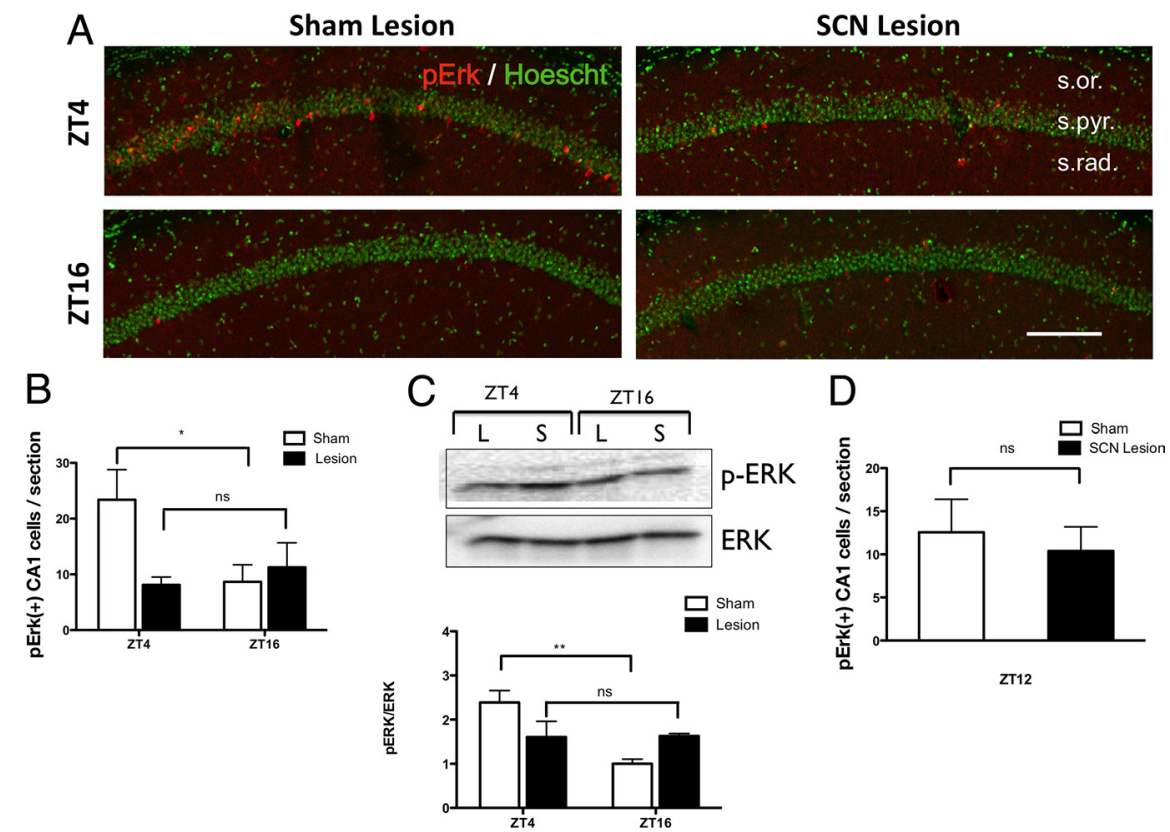

Figure 7. The diurnal oscillation of hippocampal MAPK activity is dependent upon the SCN. A, Immunodetection of pERKpositive cells (red), Hoechst (green), in the CA1 region of the hippocampus in SCN-lesioned and sham-lesioned mice at two time points, ZT4 and ZT16. Scale bar, $120 \mu \mathrm{m}$. B, Quantification of the immunohistochemical staining of pERK level in the CA1 region of the hippocampus. The values of each group were expressed as average number of $\mathrm{pERK}$-positive cells per section to the sham group killed at ZT4. ${ }^{*} p<0.05$, two-way ANOVA, Bonferroni's post hoc test. Values are expressed as means \pm SEM, $n=8-12$ per group. C, Western blot analysis of SCN-lesioned and sham-lesioned mice at ZT4 and ZT16. Western blots show pooled samples, and the graph shows results from individual animals; $n=5-6$ per group, two-way ANOVA, ${ }^{* *} p<0.01$, mean values expressed $\pm S E M$. D, Quantification of the number of pERK-immunopositive cells per section in the CA1 pyramidal layer of the hippocampus from sham- and SCN-lesioned mice at ZT12.

hibit a preference for either object (Fig. 5B). When tested $10 \mathrm{~min}$ after training, one of the original objects was replaced by a novel object, object B. During testing, both sham-lesioned and SCNlesioned mice showed a preference for the new object, indicating short-term memory for the familiar object, A1 ${ }^{* *} p=0.0079$ for
SCN lesion, and ${ }^{\star} p=0.049$ for sham control, two-tailed $t$ test) (Fig. $5 C)$. Neither sham-lesioned nor SCN-lesioned mice showed $24 \mathrm{~h}$ memory for novel objects. These data indicate that the short-term memory for novel objects does not depend upon the SCN. SCN-lesioned animals also exhibited normal open field activity and thigmotaxis in a

\section{Oscillation of adenylyl cyclase activity in the hippocampus} depends on the SCN

Activation of MAPK activity and its nuclear translocation during oscillation in the hippocampus, which is absent in memorydeficient AC1/8 double-mutant mice (Eckel-Mahan et al., yl cyclase activity in the hippocampus. Basal, forskolin-stimulated, and calcium-stimulated adenylyl cyclase activities in hippocamdifference was observed in lesioned animals at these two time points (Fig. $6 A-C$ ). Pairwise comparisons confirmed that the sham-lesioned group showed significantly higher adenylyl cyclase activities at circadian time point ZT4 versus ZT16 under basal condition $[31.46 \pm 1.37$ vs $19.71 \pm 1.087 \mathrm{pmol} \mathrm{cAMP} / \mathrm{min} / \mathrm{mg}$ protein $\left(F_{(1,11)}, p=0.002,{ }^{* *} p<0.001\right.$, twoway ANOVA)], under calcium-stimulated condition $[108.2 \pm 7.32$ vs $76.46 \pm 3.86$ $\mathrm{pmol} \mathrm{cAMP} / \mathrm{min} / \mathrm{mg}$ protein $\left(F_{(1,11)}, p=\right.$ $0.0379,{ }^{\star} p<0.05$, two-way ANOVA)], and under forskolin-stimulated condition $[182.4 \pm 6.64$ vs $115.1 \pm 1.97 \mathrm{pmol}$ $\mathrm{cAMP} / \mathrm{min} / \mathrm{mg}$ protein, $\left(F_{(1,11)}, p=\right.$ $0.0001,{ }^{* *} p<0.001$, two-way ANOVA)]. In contrast, no significant difference was observed in the SCN-lesioned group between the two circadian time points, ZT4 versus ZT16, under basal condition $(31.61 \pm 1.536$ vs $31.44 \pm 1.108 \mathrm{pmol}$ $\mathrm{cAMP} / \mathrm{min} / \mathrm{mg}$ protein), calcium-stimulated condition $(107.0 \pm 2.94$ vs $98.72 \pm 3.44 \mathrm{pmol} \mathrm{cAMP} / \mathrm{min} / \mathrm{mg}$ protein), and forskolin-stimulated condition $(186.5 \pm 7.29$ vs $192.7 \pm 3.74 \mathrm{pmol}$ $\mathrm{cAMP} / \mathrm{min} / \mathrm{mg}$ protein).

\section{Lesioning of the SCN impairs oscillations of hippocampal \\ MAPK activity}

Since the persistence of contextual memory depends upon an oscillation of MAPK activity in the hippocampus (Eckel-Mahan et al., 2008), we also measured MAPK activity in the hippocampus from shamlesioned and SCN-lesioned mice. Fixed brain slices were subjected to immunohistochemical assays, and the number of pERK-positive cells in the CA1 region was compared between sham- and SCN-lesion groups at ZT4 and ZT16 (Fig. 7A). Shamlesioned animals showed normal circadian oscillation of pERK in 
area CA1 of the hippocampus, consistent with previous data (Eckel-Mahan et al., 2008). The levels of p-ERK peaked at ZT4 and troughed at ZT16 for the sham-lesion group $\left(F_{(1,29)}, p=\right.$ $0.023,{ }^{\star} p<0.05$, two-way ANOVA) (Fig. $7 B$ ). In contrast, no significant difference in the amount of p-ERK-positive cells was observed in SCN-lesioned mice between ZT4 and ZT16 (Fig. 7B). This was also demonstrated by Western blot analysis for pERK levels in sham- and SCN-lesioned mice (Fig. $7 C$ ). In a separate experiment, we addressed the possibility that SCN lesioning phaseshifted the oscillation of pERK rather than destroyed it, by examining another time point, ZT12 (Fig. 7D). The number of pERKimmunopositive cells was similar between lesioned and control mice at ZT12, and it was still lower than the number at ZT4 in sham control mice. This indicates that the oscillation of pERK in SCNlesioned animals was destroyed and not just phase shifted. Collectively, these data suggest that circadian oscillations of $\mathrm{pERK}$ in the CA1 region of the hippocampus are dependent upon the SCN.

\section{Discussion}

Recent data indicate that the maintenance of hippocampusdependent memory depends on the oscillation of adenylyl cyclase and MAPK activities in the hippocampus suggesting that reactivation of this memory consolidation pathway during the circadian cycle is required to maintain memory (Eckel-Mahan et al., 2008). However, a major unanswered question is whether this oscillation is intrinsic to the hippocampus or depends on the $\mathrm{SCN}$. This question arises because the hippocampus exhibits intrinsic circadian oscillations in the absence molecular basis of the functional SCN (Jilg et al., 2010). Therefore, the major objectives of this study were to determine whether ablation of the SCN in mice blocks the circadian oscillation of adenylyl cyclase and MAPK activities in the hippocampus and compromises hippocampusdependent memory. In this study, the SCN was disrupted by electrolytic lesioning with sham-lesioning used as a surgical control. Since sham-lesioning involves inserting an identical electrode to the target area without delivering current, it controls for mechanical tissue damage that may arise from insertion and penetration of the electrode through other structures of the brain.

Our data indicate that SCN-lesioning but not sham-lesioning effectively disrupts the circadian rhythm of mice, confirming the role of the SCN as the master regulator of the circadian clock (Schwartz and Zimmerman, 1991; Chang and Reppert, 2001). SCN-lesioning blocked the circadian oscillation of calciumstimulated adenylyl cyclase and MAPK activities in the hippocampus. In addition, maintenance of contextual memory and spatial memory were both inhibited by SCN-lesion. It is interesting that lesioning of the SCN of context-trained animals $2 \mathrm{~d}$ after training inhibited context memory measured 2 weeks later. Since acquisition and consolidation would have occurred before SCN lesioning, this experiment supports the hypothesis that the persistence of contextual memory is dependent upon the SCN. These data are consistent with experiments that showed that administration of MEK inhibitors to the hippocampus $2 \mathrm{~d}$ after training for context blocked the oscillation of MAPK and impaired memory for context tested 1 week after delivery of the inhibitors to the hippocampus (Eckel-Mahan et al., 2008).

The behavioral data reported in this study is consistent with other research showing that direct lesions of the SCN (Stephan and Kovacevic, 1978) phase shifting (Tapp and Holloway, 1981), or genetic manipulations rendering the SCN nonfunctional (Garcia et al., 2000) all lead to memory deficits. Our data significantly extends these observations by providing a molecular mechanism that explains why perturbation of SCN function impairs hippocampus-dependent memory. Our study indicates that SCN function is required for the circadian oscillation of adenylyl cyclase and MAPK activities in the hippocampus, an oscillation necessary for the maintenance of hippocampus-dependent memory (Eckel-Mahan et al., 2008). Even though the SCN does not directly project to the hippocampus, it projects to multiple areas including the septum that innervate hippocampal subregions (Watts and Swanson, 1987; Morin et al., 1994). Furthermore, the SCN can indirectly modulate adenylyl cyclase activity during the circadian cycle by controlling the release of melatonin from the pineal gland (Yamanaka et al., 2010). Melatonin receptors in the hippocampus are coupled to inhibition of adenylyl cyclase activity through $G_{i}$ and suppression of LTP (Wang and Storm, 2005). The inhibition of calcium-sensitive adenylyl cyclase activity by nocturnal melatonin could explain the oscillation in adenylyl cyclase and MAPK activities.

Although spatial, contextual, and passive avoidance memories were impaired by lesioning of the SCN, short-term memory for novel objects was unaffected. There has been conflicting evidence concerning the role of the hippocampus in memory for novel objects. Some studies have reported novel object recognition impairments in animals with hippocampal lesions (Clark et al., 2000; Broadbent et al., 2004) while others have reported no impairments (Winters et al., 2004; Good et al., 2007). The discrepancy in these finding may be due to differences in the size of the hippocampal lesion or behavioral procedures used in the different studies. However, a recent reexamination of this question by intrahippocampal injection of the GABA agonist muscimol indicates that the formation of object recognition memory does not depend on the hippocampus (Oliveira et al., 2010). This may explain why SCN-lesions and the loss of oscillations in MAPK activity in the hippocampus did not affect short-term memory for novel objects. Alternatively, SCN-lesioning may not generally affect short-term memory. SCN-lesioned mice learned to the find the hidden platform in the Morris water maze as fast as shamlesioned mice indicating no defect in short-term spatial memory. Furthermore, relearning of the platform position in the Morris water maze after platform reversal was unaffected by SCN lesions. This suggests that the SCN is not required for short-term hippocampus-dependent memory.

It might be argued that the effect of SCN lesioning on hippocampus-dependent memory and MAPK activity is due to sleep deprivation. Moreover, although SCN lesioning disrupts the circadian rhythm of rats, the total amount of sleep as well as REM and non-REM sleep is unaffected by lesioning of the SCN (Baker et al., 2005). In addition, SCN lesioning of BALB/c mice causes no differences in the total amount of sleep but affects sleep patterns (Ibuka et al., 1980). Consequently, we can not rule out the possibility that the effects of SCN-lesioning on circadian oscillation of MAPK activity is due to changes in sleep pattern.

In conclusion, diurnal oscillation of hippocampal MAPK and adenylyl cyclase activities is dependent on the SCN and is not an autonomous oscillation intrinsic to the hippocampus. We hypothesize that memory defects caused by lesioning of the SCN are due, at least in part, to inhibition of the diurnal oscillation of this signaling pathway in the hippocampus. Our biochemical data provides an explanation why memory may be affected by damage to the SCN or other physiological conditions that impair the circadian rhythm of animals. 


\section{References}

Athos J, Impey S, Pineda VV, Chen X, Storm DR (2002) Hippocampal CREmediated gene expression is required for contextual memory formation. Nat Neurosci 5:1119-1120.

Atkins CM, Selcher JC, Petraitis JJ, Trzaskos JM, Sweatt JD (1998) The MAPK cascade is required for mammalian associative learning. Nat Neurosci 1:602-609.

Bailey CH, Bartsch D, Kandel ER (1996) Toward a molecular definition of long-term memory storage. Proc Natl Acad Sci U S A 93:13445-13452.

Baker FC, Angara C, Szymusiak R, McGinty D (2005) Persistence of sleeptemperature coupling after suprachiasmatic nuclei lesions in rats. Am J Physiol Regul Integr Comp Physiol 289:R827-R838.

Blum S, Moore AN, Adams F, Dash PK (1999) A mitogen-activated protein kinase cascade in the CA1/CA2 subfield of the dorsal hippocampus is essential for long-term spatial memory. J Neurosci 19:3535-3544.

Bourtchuladze R, Frenguelli B, Blendy J, Cioffi D, Schutz G, Silva AJ (1994) Deficient long-term memory in mice with a targeted mutation of the cAMP-responsive element-binding protein. Cell 79:59-68.

Broadbent NJ, Squire LR, Clark RE (2004) Spatial memory, recognition memory, and the hippocampus. Proc Natl Acad Sci U S A 101:14515-14520.

Chang DC, Reppert SM (2001) The circadian clocks of mice and men. Neuron 29:555-558.

Clark RE, Zola SM, Squire LR (2000) Impaired recognition memory in rats after damage to the hippocampus. J Neurosci 20:8853-8860.

Danysz W, Wroblewski JT, Costa E (1988) Learning impairment in rats by $N$-methyl-D-aspartate receptor antagonists. Neuropharmacology 27:653-656.

Davis HP, Squire LR (1984) Protein synthesis and memory: a review. Psychol Bull 96:518-559.

Eckel-Mahan KL, Phan T, Han S, Wang H, Chan GC, Scheiner ZS, Storm DR (2008) Circadian oscillation of hippocampal MAPK activity and cAMP: implications for memory persistence. Nat Neurosci 11:1074-1082.

Fanselow MS, Poulos AM (2005) The neuroscience of mammalian associative learning. Annu Rev Psychol 56:207-234.

Garcia JA, Zhang D, Estill SJ, Michnoff C, Rutter J, Reick M, Scott K, DiazArrastia R, McKnight SL (2000) Impaired cued and contextual memory in NPAS2-deficient mice. Science 288:2226-2230.

Gerstner JR, Yin JC (2010) Circadian rhythms and memory formation. Nat Rev Neurosci 11:577-588.

Good MA, Barnes P, Staal V, McGregor A, Honey RC (2007) Context- but not familiarity-dependent forms of object recognition are impaired following excitotoxic hippocampal lesions in rats. Behav Neurosci 121:218-223.

Granados-Fuentes D, Prolo LM, Abraham U, Herzog ED (2004a) The suprachiasmatic nucleus entrains, but does not sustain, circadian rhythmicity in the olfactory bulb. J Neurosci 24:615-619.

Granados-Fuentes D, Saxena MT, Prolo LM, Aton SJ, Herzog ED (2004b) Olfactory bulb neurons express functional, entrainable circadian rhythms. Eur J Neurosci 19:898-906.

Hastings MH, Reddy AB, Maywood ES (2003) A clockwork web: circadian timing in brain and periphery, in health and disease. Nat Rev Neurosci $4: 649-661$

Ibuka N, Nihonmatsu I, Sekiguchi S (1980) Sleep-wakefulness rhythms in mice after suprachiasmatic nucleus lesions. Waking Sleeping 4:167-173.

Jilg A, Lesny S, Peruzki N, Schwegler H, Selbach O, Dehghani F, Stehle JH (2010) Temporal dynamics of mouse hippocampal clock gene expression support memory processing. Hippocampus 20:377-388.

Li W, Cui Y, Kushner SA, Brown RA, Jentsch JD, Frankland PW, Cannon TD, Silva AJ (2005) The HMG-CoA reductase inhibitor lovastatin reverses the learning and attention deficits in a mouse model of neurofibromatosis type 1. Curr Biol 15:1961-1967.

Moore RY, Eichler VB (1972) Loss of a circadian adrenal corticosterone rhythm following suprachiasmatic lesions in the rat. Brain Res 42:201-206.
Morin LP, Goodless-Sanchez N, Smale L, Moore RY (1994) Projections of the suprachiasmatic nuclei, subparaventricular zone and retrochiasmatic area in the golden hamster. Neuroscience 61:391-410.

Morris RG, Anderson E, Lynch GS, Baudry M (1986) Selective impairment of learning and blockade of long-term potentiation by an $N$-methyl-Daspartate receptor antagonist, AP5. Nature 319:774-776.

Oliveira AM, Hawk JD, Abel T, Havekes R (2010) Post-training reversible inactivation of the hippocampus enhances novel object recognition memory. Learn Mem 17:155-160.

Pittenger C, Huang YY, Paletzki RF, Bourtchouladze R, Scanlin H, Vronskaya S, Kandel ER (2002) Reversible inhibition of CREB/ATF transcription factors in region CA1 of the dorsal hippocampus disrupts hippocampusdependent spatial memory. Neuron 34:447-462.

Reppert SM, Weaver DR (2001) Molecular analysis of mammalian circadian rhythms. Annu Rev Physiol 63:647-676.

Rosbash M, Hall JC (1989) The molecular biology of circadian rhythms. Neuron 3:387-398.

Schwartz WJ, Zimmerman P (1991) Lesions of the suprachiasmatic nucleus disrupt circadian locomotor rhythms in the mouse. Physiol Behav 49:1283-1287.

Selcher JC, Atkins CM, Trzaskos JM, Paylor R, Sweatt JD (1999) A necessity for MAP kinase activation in mammalian spatial learning. Learn Mem 6:478-490.

Sindreu CB, Scheiner ZS, Storm DR (2007) $\mathrm{Ca}^{2+}$-stimulated adenylyl cyclases regulate ERK-dependent activation of MSK1 during fear conditioning. Neuron 53:79-89.

Stephan FK, Kovacevic NS (1978) Multiple retention deficit in passive avoidance in rats is eliminated by suprachiasmatic lesions. Behav Biol 22:456-462.

Stephan FK, Zucker I (1972) Circadian rhythms in drinking behavior and locomotor activity of rats are eliminated by hypothalamic lesions. Proc Natl Acad Sci U S A 69:1583-1586.

Tapp WN, Holloway FA (1981) Phase shifting circadian rhythms produces retrograde amnesia. Science 211:1056-1058.

Tsien JZ, Huerta PT, Tonegawa S (1996) The essential role of hippocampal CA1 NMDA receptor-dependent synaptic plasticity in spatial memory. Cell 87:1327-1338.

Tully T, Preat T, Boynton SC, Del Vecchio M (1994) Genetic dissection of consolidated memory in Drosophila. Cell 79:35-47.

Turek FW (1981) Are the suprachiasmatic nuclei the location of the biological clock in mammals? Nature 292:289-290.

Vorhees CV, Williams MT (2006) Morris water maze: procedures for assessing spatial and related forms of learning and memory. Nat Protoc $1: 848-858$.

Wang H, Storm D (2005) $\mathrm{Ca}^{2+}$-stimulated adenylyl cyclases and hippocampal neuroplasticity. Cell Science Rev 2:73-91.

Wang LM, Dragich JM, Kudo T, Odom IH, Welsh DK, O’Dell TJ, Colwell CS (2009) Expression of the circadian clock gene Period2 in the hippocampus: possible implications for synaptic plasticity and learned behaviour. ASN Neuro 1:e00012.

Watts AG, Swanson LW (1987) Efferent projections of the suprachiasmatic nucleus: II. Studies using retrograde transport of fluorescent dyes and simultaneous peptide immunohistochemistry in the rat. J Comp Neurol 258:230-252.

Winters BD, Forwood SE, Cowell RA, Saksida LM, Bussey TJ (2004) Double dissociation between the effects of peri-postrhinal cortex and hippocampal lesions on tests of object recognition and spatial memory: heterogeneity of function within the temporal lobe. J Neurosci 24:5901-5908.

Yamanaka Y, Suzuki Y, Todo T, Honma K, Honma S (2010) Loss of circadian rhythm and light-induced suppression of pineal melatonin levels in Cry1 and Cry2 double-deficient mice. Genes Cells 15:1063-1071. 